\title{
Effects of single-session repetitive judgments on magnitude estimation scales for lingual vibrotactile sensation
}

\author{
LINDA PETROSINO \\ University of North Carolina, Chapel Hill, North Carolina \\ and \\ DONALD FUCCI and DANIEL HARRIS \\ Ohio University, Athens, Ohio
}

\begin{abstract}
The human tongue, because it is mucosal in nature and housed within the oral cavity, presents a unique set of problems for suprathreshold vibrotactile magnitude scaling. It was hypothesized that the ideal response mode when testing the lingual structure would entail a single set of stimulus-level judgments to be made by a subject so that fatigue effects and overall subject discomfort associated with lingual vibrotactile suprathreshold testing could be reduced. The purpose of the present investigation was to study the possible effects that single-session repetitive judgments would have on lingual vibrotactile magnitude estimation outcome. Twenty subjects (M age $=23.25$ years) participated in a single-session magnitude estimation paradigm which required them to make three magnitude estimation judgments at each of nine stimulus intensity levels. The three data runs were statistically analyzed using a multivariate mixed-model analysis of variance. There appeared to be no statistical differences between the three magnitude scaling runs at any of the nine sensation levels, whether they were compared separately or in all possible combinations, when alpha was set at .05 . The results are discussed as being supportive of the possibility that a single set of response judgments can be employed in lingual vibrotactile magnitude scaling. Factors associated with the single-response set, such as ability to run more subjects in less time, better assurance of open-ended responses from subjects, and the increased likelihood of subject response spontaneity are also discussed.
\end{abstract}

In 1957, S. S. Stevens reported that a power function law successfully described the relationship between subjective magnitude and the physical intensity of stimuli applied to various sensory systems. Stevens (1961) performed a series of studies relating subjective responses to vibratory stimulus intensities applied to the fingertip. Subsequently, several researchers have studied some of the possible parameters that can affect suprathreshold vibrotactile power functions. Such parameters as psychophysical scaling method, size of the skin contactor, use of a rigid surface to surround the contactor, body site of stimulation, frequency at which scaling is performed, and signal duration have been shown to have an effect on the magnitude functions obtained (Verrillo \& Capraro, 1975; Verrillo \& Chamberlain, 1972; Verrillo, Fraioli, \& Smith, 1969; Verrillo \& Smith, 1976; Zwislocki \& Goodman, 1980). In all of these subsequent studies, the test sites for stimulation were selected from three body locations: the distal pad of the middle finger, the thenar eminence of the hand, and the volar forearm.

D. Fucci's mailing address is: School of Hearing and Speech Sciences, Lindley Hall, Ohio University, Athens, OH 45701.
In 1983, a series of studies was initiated in which psychophysical scaling technique was used in vibrotactile testing of the dorsal surface of the human tongue (Fucci, Harris, \& Petrosino, 1983a, 1983b, 1984; Fucci \& Petrosino, 1983a, 1983b, 1983c; Fucci, Petrosino, \& Harris, 1983, 1984; Petrosino, Fucci, \& Harris, 1984). Many of the parameters studied for the fingertip, thenar eminence, and volar forearm were reexamined, and alterations in scaling procedure were made in deference to the uniqueness of the lingual structure. The lingual structure, unlike the sites commonly tested on the hand and arm, is mucosal in nature (Grossman \& Hattis, 1967). The protrusion of the tongue for testing can create a sensory fatigue effect and overall subject discomfort that has to be accounted for in the psychophysical scaling paradigm being utilized.

Initially, the method of magnitude production was chosen for lingual vibrotactile scaling (Fucci \& Petrosino, 1983a, 1983b, 1983c). It was selected because it appeared to be more stable than the method of magnitude estimation (Zwislocki \& Goodman, 1980). Pilot work also showed that magnitude production scaling could be managed on the tongue without serious restrictions being placed on stimulus intensity range and without subjects' 
becoming fatigued beyond the point of reliable testing. A paradigm developed by Hellman and Zwislocki (1963) was adopted in which subjects were asked to scale the range of stimuli three times in one session. The first run was considered practice and was discarded; the means of the second and third runs were considered as the subjects' magnitude production scaling responses. Appropriate criticisms have been made that the magnitude production procedure developed for lingual vibrotactile testing did not provide enough scaling levels (six judgment levels were used), and that the numbers used in scaling were derived by the experimenters rather than from the magnitude estimation responses of the subjects.

In 1984, Fucci, Harris, and Petrosino adapted a psychophysical scaling paradigm for the tongue which consisted of magnitude estimation scaling followed by magnitude production scaling based on the subjects' magnitude estimation responses (Hellman \& Zwislocki, 1963). The judgment levels were extended from six to nine for both the magnitude estimation and magnitude production procedures. In order to reduce subject fatigue resulting from the increase in the number of judgment levels, it became necessary to eliminate the practice run suggested by Hellman and Zwislocki. Two runs for each of the nine judgment levels were conducted, and the means of these runs were accepted as a subject's magnitude scaling responses. This procedure was followed for both magnitude estimation and magnitude production, and did not appear to have a noticeable effect on overall lingual vibrotactile scaling results (Fucci, Harris, \& Petrosino, 1984).

The ideal response mode for vibrotactile psychophysical scaling on the lingual dorsal surface would be one requiring a single set of stimulus level judgments to be made by a subject. This procedural modification would reduce the possibility of fatigue effects during lingual vibrotactile testing, permit an increase in the number of response levels required of a subject, and allow data collection from more_subjects within the same amount of time. The purpose of the present investigation was to study the possible effects of single-session repetitive judgments in lingual vibrotactile magnitude scaling procedure. Specifically, the study was designed to investigate possible differences in magnitude estimation scaling values across three sets of judgments obtained in the same experimental session.

\section{METHOD}

The vibrotactile instrumentation consisted of a stimulus unit and a measurement unit. The stimulus unit was composed of a sinewave generator, an experimenter-controlled 2 -dB-step variable attenuator, two universal timers, an audio amplifier, a power amplifier, a preamplifier, and an electromagnetic minivibrator with a probe-contactor extension. The pulsed vibratory signal generated had a $50 \%$ duty cycle (on $500 \mathrm{msec}$ and off $500 \mathrm{msec}$ ), with a riseand-decay time of $100 \mathrm{msec}$. The signal frequency was set at $250 \mathrm{~Hz}$. The measurement unit included an accelerometer, a cathode follower, a microphone amplifier, and a voltmeter. A narrow-band noise generator was used to present auditory masking at $70 \mathrm{~dB}$ HTL to the subjects to prevent them from hearing the vibratory stimulus. A detailed description of the instrumentation can be found in an article by Fucci, Petrosino, Wallace, and Small (1982).

Twenty subjects, 4 men and 16 women, aged 17-36 years (M age $=23.25$ years), were selected randomly from courses in speech and hearing sciences. All subjects had normal speech and hearing and reported no known sensory and/or motor impairments. None of the subjects were experienced in vibrotactile testing or psychophysical scaling technique. All 20 subjects were treated in the same manner. During a single testing session, each subject was seated in an adjustable chair and positioned so that the dorsal surface of the tongue was in contact with the lower side of a rigidly mounted plastic disk. A hole in the center of the disk provided access to the lingual dorsal surface for the probe-contactor extension of the vibrator. The contactor had an area of $.128 \mathrm{~cm}^{2}$ and there was a 1-mm gap between the contactor and the plastic disk.

Lingual thresholds of sensitivity were established for each subject through use of the method of limits. The psychophysical method of magnitude estimation was used to obtain suprathreshold magnitude functions for the tongue of each subject (Stevens, 1955). To minimize possible biases in the results, no reference standard was employed in the suprathreshold scaling task (Hellman \& Zwislocki, 1963). The subjects were required to assign numbers to a randomly presented series of nine stimulus intensities ranging from 2 to $40 \mathrm{~dB}$ SL. Each subject was instructed to "think of a number that matches the strength of the vibration." Whole numbers, decimals, and fractions were indicated as permissible selections (Zwislocki \& Goodman, 1980). The subjects were encouraged to be spontaneous in selecting numbers and to judge each stimulus without reference to those previously presented. Three runs of the nine stimulus intensities were performed for each subject. Means for the 20 subjects were derived from their numerical responses to each of the nine stimulus intensities for each of the three scaling runs.

\section{RESULTS AND DISCUSSION}

The mean numerical responses of the 20 subjects for each of three magnitude scaling runs are shown in Table 1 . The subject response data were analyzed using a multivariate mixed-model analysis of variance (Bock, 1975). All statistical tests were conducted with an a priori alpha level of .05 . No overall statistical differences were found between the subject responses for the three data runs at any of the nine sensation levels. No statistical differences were found when the means of Runs 1 and 2, Runs 2 and 3, and Runs 1 and 3 were compared with each other at any of the nine sensation levels. There appeared to be no statistical differences between the three magnitude scaling runs at any of the nine sensation levels, whether they were compared separately or in all possible combinations.

The scaling paradigm developed by Hellman and Zwislocki (1963) appears to have resulted from their observation that inexperienced subjects tended to select numbers for the first scaling run that were higher in the lower sensation levels and lower in the higher sensation levels than numbers selected on succeeding runs performed within the same test session. Zwislocki and Goodman (1980) noted a similar pattern of scaling behavior in a group of inexperienced subjects, who judged the loudness of 20 - 
msec bursts of a $1000-\mathrm{Hz}$ tone. The authors suggested that experience gained from the first run may have enabled the subjects to establish stable scaling behavior by the second run. The subjects were able to perform additional runs providing numerical responses at all judgment levels that were similar to those of the second run.

It can be seen in Table 1 that, in the present study, the mean numerical responses for lingual vibrotactile magnitude estimation follow the trends reported by Hellman and Zwislocki (1963) and Zwislocki and Goodman (1980). The lower sensation levels for Run 1 show mean numerical responses that are higher than those for Runs 2 and 3 , and the higher sensation levels for Run 1 show mean numerical responses that are lower than those for Runs 2 and 3. The numerical responses for Runs 2 and 3 visually appear to be compatible at all sensation levels. Statistical analysis, however, showed the visual differences noted between Run 1 and Runs 2 and 3 to be nonsignificant at the .05 alpha level for all of the nine sensation levels.

Figure 1 shows a graph of the mean numerical responses of the 20 subjects for each of the three magnitude scaling runs. The three plotted curves are compatible with each other and with curves that have been generated for the tongue previously (Fucci, Harris, \& Petrosino, 1984). The curves are somewhat different on the upper and lower ends than those generated for the hand (fingertip and thenar eminence) (Verrillo \& Chamberlain, 1972). It appears, from the curves in Figure 1 , that asymptotic scaling behavior is demonstrated for the tongue at the upper stimulus intensity levels, which are much greater than those used for the hand. The lower end of the magnitude estimation curves for the tongue appears to show less consistency than those for the hand. This difference may be the result of sensitivity differences between the tongue and the hand. A typical range of thresholds for the fingertip and thenar eminence of the hand would be -14 to $-10 \mathrm{~dB}$ re $1 \mu$ peak (Verrillo \& Chamberlain, 1972), whereas a typical range of sensitivity for the lingual dorsal surface would be 1.0 to $2.5 \mathrm{~dB}$ re $1 \mu$ peak (Fucci \& Crary, 1979).

Table 1

Mean Numerical Responses of 20 Subjects for Three Magnitude Estimation Scaling Runs Performed on the Dorsal Surface of the Tongue

\begin{tabular}{crrr}
\hline & \multicolumn{3}{c}{ Means } \\
\cline { 2 - 4 } Sensation Level* & Run 1 & Run 2 & Run 3 \\
\hline 2 & .69 & .31 & .22 \\
6 & 1.12 & .47 & .29 \\
10 & 1.13 & .49 & .57 \\
14 & 2.08 & 1.11 & 1.52 \\
20 & 4.44 & 2.92 & 3.60 \\
24 & 6.25 & 6.14 & 6.78 \\
30 & 11.04 & 11.34 & 11.99 \\
34 & 11.79 & 13.94 & 16.50 \\
40 & 11.82 & 16.66 & 17.92 \\
\hline
\end{tabular}

*In decibels.

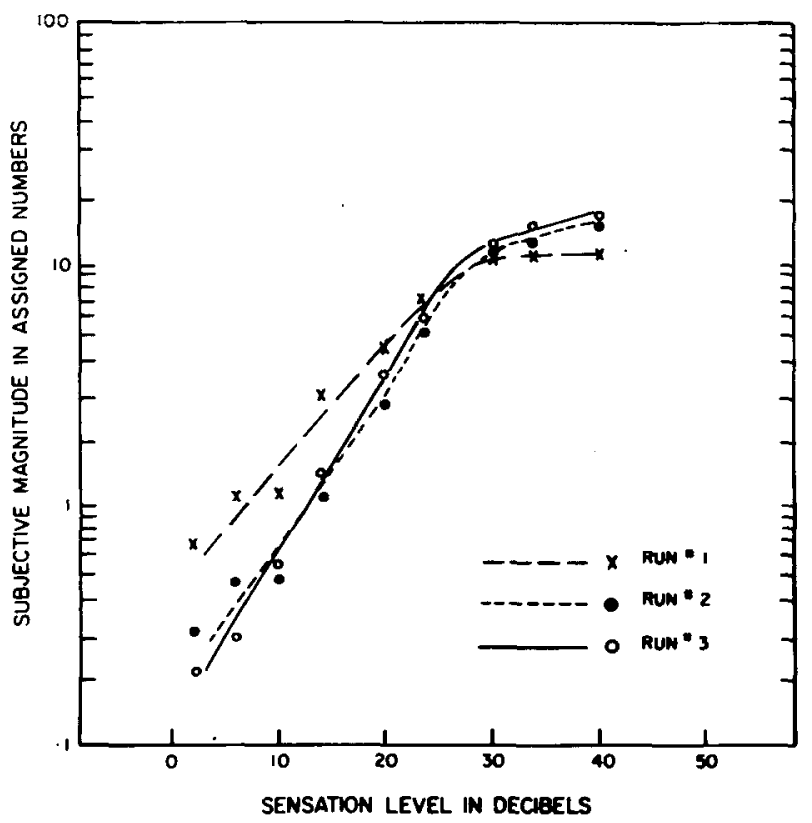

Figure 1. Magnitude estimation functions for three scaling runs performed on the dorsal surface of the tongue.

When viewed in the framework of previous paradigms that used three sets of judgments in the same experimental session (Hellman \& Zwislocki, 1963; Zwislocki \& Goodman, 1980), the results of the present study appear to indicate that the number of judgment runs to be used in a magnitude scaling experiment may depend on the purpose of the investigation and the conditions necessary for sampling behavior. For example, a study of the possible "absolute" response values (Zwislocki \& Goodman, 1980 ) for the scaling of a particular stimulus may require a practice run in order to obtain samples of more consistent intrasubject judgments. All human sensory-perceptual systems have their limits of durability, however, and there is a point where repetitive scaling judgments will lead to system fatigue and increased response variability (Stevens \& Greenbaum, 1966). In a study concerned with initial psychophysical responses to a particular stimulus, a single data run may be the ideal choice because additional runs within the same session do not appear to add information and, in fact, may result in fatigue effects in the subjects.

Several researchers seem to favor the psychophysical scaling paradigm that does not include repetitive subject responses within a single testing session. Stevens and Greenbaum (1966) suggest that in a given experiment, the increment of new information decreases quite rapidly after the first series of judgments is made. They indicate that brief experiments yield relatively more information than protracted ones. Teghtsoonian and Teghtsoonian $(1971,1983)$ report that repeated judging in the same test session allows memorization and learning to take place, and that one set of judgments can constrain a subsequent 
set when the interval between them is short. If several judgments are made at each scaling level within a single test session, the constraint may be extended as much as $24 \mathrm{~h}$ (Teghtsoonian \& Teghtsoonian, 1971, 1983). These researchers suggest, therefore, that if experimenters want independent scaling responses from their subjects, at least 1 day should elapse between sessions and subjects should judge a particular stimulus value only once per session. Repeated judgments within a single session will lead to increased response stability, but the amount of new information in second and succeeding judgment sets is small and the additional time commitment is not justified (Teghtsoonian \& Teghtsoonian, 1971, 1983). Eisler (1982) suggests that a critical element in both magnitude estimation and magnitude production is a paradigm that permits openended responses with minimal constraints imposed upon the subjects by the experimenter. If memory and learning is encouraged through repetitive response judgments, then subject spontaneity is eliminated (Teghtsoonian \& Teghtsoonian, 1983). Warren (1981) indicates that one way of minimizing contextual effects when performing psychophysical intensity judgment experiments is to use only first judgments from large groups of subjects.

Lingual vibrotactile suprathreshold scaling research appears to lend itself favorably to the scaling paradigm that makes use of a single set of response judgments. The lingual structure presents some unique testing problems which are minimized by the single-response-set approach. For example, there is less opportunity for the system fatigue and overall subject discomfort that is associated with the tongue's being held for extended periods in test position. The other positive factors of being able to run more subjects in less time, receiving open-ended responses from subjects with minimal experimenter constraints, and assurance of subject response spontaneity add to the attractiveness of this approach. Future research in psychophysics devoted to the study of conditions required for response accuracy and conditions decreasing response bias is needed to further refine the methods used for sampling subjective magnitude scaling behavior.

\section{REFERENCES}

Bock, R. D. (1975). Multivariate statistical methods in behavioral research. New York: McGraw-Hill.

EISLER, H. (1982). On the nature of subjective scales. Scandinavian Journal of Psychology, 23, 161-171.

FUCCI, D., CRARY, M. (1979). Oral vibrotactile sensation and perception: State of the art. In N. Lass (Ed.), Speech and language: Advances in basic research and practice (Vol. 2). New York: Academic Press.

Fucci, D., Harris, D., \& Petrosino, L. (1983a). The effect of contactor area on vibrotactile magnitude function exponents for the tongue and hand. Bulletin of the Psychonomic Society, 21, 400-402.

FucCI, D., Harris, D., \& Petrosino, L. (I983b). Stability of magnitude production scales for lingual vibrotactile sensation. Joumal of Perceptual \& Motor Skills, 57, 499-504.
FucCI, D., Harris, D., \& Petrosino, L. (1984). Sensation magnitude scales for vibrotactile stimulation of the tongue and thenar eminence. Journal of Perceptual \& Motor Skills, 58, 843-848.

Fucci, D. , \& PeTrosino, L. (1983a). Lingual vibrotactile sensation magnitudes: Comparison of suprathreshold responses in men and women. Perception \& Psychophysics, 33, 93-95.

Fucci, D., \& Petrosino, L. (1983b). Lingual vibrotactile sensation magnitudes: Comparison of suprathreshold responses for three different age ranges. Journal of Perceptual \& Motor Skills, 57, 31-38.

Fucci, D., \& PETrosino, L. (1983c). Lingual vibrotactile sensation magnitudes: Comparison of suprathreshold responses for the tongue and hand. Journal of the Acoustical Society of America, 74, 351-353.

Fucci, D., Petrosino, L., \& Harris, D. (1983). Influence of scaling range on vibrotactile power function exponents for the tongue and hand. Journal of Perceptual \& Motor Skills, 57, 642.

Fucci, D., Petrosino, L., \& HARrIs, D. (1984). Stability of magnitude production scales for oral and nonoral vibrotactile mechanoreceptive systems. Journal of Perceptual \& Motor Skills, 58, 691-697.

Fucci, D., Petrosino, L., Wallace, D., \& Small, L. (1982). Modification of instrumentation for research on lingual vibrotactile sensitivity: Elimination of the tongue clamping procedure. Review of Scientific Instruments, 53, 1294-1296.

Grossman, R. C., \& Hattis, B. F. (1967). Oral mucosal sensory innervation and sensory experience. In J. F. Bosma (Ed.), Symposium on oral sensation and perception. Springfield, IL: Thomas.

Hellman, R. P., \& Zwislocki, J. (1963). Monaural loudness function at $1000 \mathrm{cps}$ and interaural summation. Journal of the Acoustical Society of America, 35, 856-865.

Petrosino, L., Fucci, D., \& Harris, D. (1984). Threshold influences on vibrotactile magnitude production scaling. Bulletin of the Psychonomic Society, 22, 139-141.

Stevens, S. S. (1955). The measurement of loudness. Journal of the Acoustical Society of America, 27, 815-820.

Stevens, S. S. (1957). On the psychophysical law. Psychological Review, 64, 153-181.

STEVENS, S. S. (1961). The psychophysics of sensory function. In W. A. Rosenblith (Ed.), Sensory communication. New York: Wiley.

Stevens, S. S., \& Greenbaum, H. B. (1966). Regression effect in psychophysical judgment. Perception \& Psychophysics, 1, 439-446.

Teghtsoonian, M., \& Teghrsoonian, R. (1971). How repeatable are Stevens's power law exponents for individual subjects? Perception \& Psychophysics, 10, 147-149.

Teghtsoonian, M., \& Teghtsoonian, R. (1983). Consistency of individual exponents in cross-modal matching. Perception \& Psychophysics, 33, 203-214.

VERRILLO, R. T., \& CAPRARo, A. (1975). Effects of stimulus frequency on subjective vibrotactile magnitude functions. Perception \& Psychophysics, 17, 91-96.

Verrillo, R. T., Chamberlain, S. C. (1972). The effect of neural density and contactor surround on vibrotactile sensation magnitude. Perception \& Psychophysics, 11, 117-120.

Verrillo, R. T., Fraioli, A. J., \&Mith, R. L. (1969). Sensation magnitude of vibrotactile stimuli. Perception \& Psychophysics, 6, 366-372.

VERRILLO, R. T., \&MITH, R. (1976). Effects of stimulus duration on vibrotactile sensation magnitude. Bulletin of the Psychonomic Society, 8, 112-114.

WARREN, R. M. (1981). Measurement of sensory intensity. Behavioral \& Brain Sciences, 4, 175-223.

ZWISLOCK1, J., GOODMAN, D. (1980). Absolute scaling of sensory magnitudes: A validation. Perception \& Psychophysics, 28, 28-38.

(Manuscript received November 9, 1984; revision accepted for publication February 10, 1985.) 